\title{
High Frequency of $\beta$-Catenin Mutations in Mouse Hepatocellular Carcinomas Induced by a Nongenotoxic Constitutive Androstane Receptor Agonist
}

\author{
Sandra Mattu, ${ }^{*}$ Christian Saliba ${ }^{\dagger}$ Pia Sulas, ${ }^{*}$ Patrizia Zavattari, ${ }^{*}$ Andrea Perra, ${ }^{*}$ Marta A. Kowalik, ${ }^{*}$ Satdarshan P. Monga, ${ }^{\ddagger}$ and \\ Amedeo Columbano*
}

From the Department of Biomedical Sciences, ${ }^{*}$ University of Cagliari, Cagliari, Italy; the Centre of Molecular Medicine and Biobanking, ${ }^{\dagger}$ University of Malta, Msida, Malta; and the Department of Pathology, ${ }^{\ddagger}$ University of Pittsburgh Medical Center, University of Pittsburgh, School of Medicine, Pittsburgh, Pennsylvania

\author{
Accepted for publication \\ July 24, 2018. \\ Address correspondence to \\ Amedeo Columbano, Ph.D., \\ Department of Biomedical \\ Sciences, Unit of Oncology and \\ Molecular Pathology, \\ University of Cagliari, Via \\ Porcell 4, Cagliari 09124, \\ Italy. E-mail: columbano@ \\ unica.it.
}

\begin{abstract}
Activation of Wnt/ $\beta$-catenin signaling is frequent in human and rodent hepatocarcinogenesis. Although in mice the tumor-promoting activity of agonists of constitutive androstane receptor (CAR) occurs by selection of carcinogen-initiated cells harboring $\beta$-catenin mutations, the molecular alterations leading to hepatocellular carcinoma (HCC) development by the CAR agonist 1,4-bis[2-(3,5-dichloropyridyloxy)] benzene (TCP) in the absence of genotoxic injury are unknown. Here, we show that CAR activation per se induced $\mathrm{HCC}$ in mice and that $91 \%$ of them carried $\beta$-catenin point mutations or large in-frame deletions/exon skipping targeting Ctnnb1 exon 3. Point mutations in HCCs induced by TCP alone displayed different nucleotide substitutions compared with those found in HCCs from mice pretreated with diethylnitrosamine. Moreover, unlike those occurring in HCCs from diethylnitrosamine + TCP mice, they did not result in increased expression of $\beta$-catenin target genes, such as Glul, Lgr5, Rgn, Lect2, Tbx3, Axin2, and Cond1, or nuclear translocation of $\beta$-catenin compared with the control liver. Remarkably, in the nontumoral liver tissue, chronic CAR activation led to down-regulation of these genes and to a partial loss of glutamine synthetase-positive hepatocytes. These results show that, although chronic CAR activation per se induces HCCs carrying $\beta$-catenin mutations, it concurrently down-regulates the Wnt/ $\beta$-catenin pathway in nontumoral liver. They also indicate that the relationship between CAR and $\beta$-catenin may be profoundly different between normal and neoplastic hepatocytes. (Am J Pathol 2018, 188: 2497-2507; https://doi.org/10.1016/j.ajpath.2018.07.022)
\end{abstract}

$\beta$-Catenin is a component of adherens junctions in epithelial cells, and it exerts a crucial role in intercellular adhesion through its association with $\boldsymbol{\alpha}$-catenin and E-cadherin. ${ }^{1-3}$ The disruption of this complex impairs epithelial tissue integrity, leading to cell motility and invasion. ${ }^{4}$

In addition to its adhesive function, $\beta$-catenin is a critical downstream effector of Wnt signaling pathway, where it mediates a nuclear response after the binding of Wnt proteins at the plasma membrane. In unstimulated adult cells, when the Wnt pathway is inactive, $\beta$-catenin is recruited into a destruction complex with Axin, adenomatous polyposis coli, casein kinase1, and glycogen synthase kinase-3 $\beta$. Casein kinase 1 phosphorylates $\beta$-catenin at $\mathrm{S}$ residue of codon 45 that triggers the phosphorylation by glycogen synthase kinase-3 $\beta$ at residues S33, S37, and
$\mathrm{T} 41,{ }^{5}$ which results in $\beta$-catenin recognition by the $\beta$-transducing repeat-containing protein for ubiquitination and subsequent proteasomal degradation. ${ }^{6}$

The disruption of the complex releases $\beta$-catenin and results in its cytoplasmic accumulation and nuclear translocation, whereby it functions through the transcriptional regulation of its target genes. ${ }^{7} \beta$-Catenin target genes are involved in embryonic and tissue development, cellular differentiation, survival, regeneration, self-renewal, and oncogenic transformation of several cell types..$^{7-12}$ In the

Supported by Associazione Italiana Ricerca sul Cancro (AIRC) grant IG15279 and Fondazione di Sardegna (A.C.). S.M. and M.A.K. are recipients of AIRC and Fondazione Umberto Veronesi fellowships, respectively.

Disclosures: None declared. 
liver, many $\beta$-catenin target genes have been identified, such as glutamine synthetase (GS; also known as $G L U L)$, the glutamate transporter $(G L T 1)$, ornithine aminotransferase $(O A T){ }^{13}$ an orphan G-protein-coupled receptor (GPR49; also known as $L G R 5),{ }^{14}$ a chemokine-like protein $L E C T 2,{ }^{15}$ Cyclin D1 $(C C N D 1),{ }^{16}$ regucalcin $(R G N),{ }^{17}$ and T-Box3 (TBX3) ${ }^{18}$ Activation of Wnt signaling because of different mechanisms that range from mutations in CTNNB1, AXIN1, AXIN2, and others ${ }^{19}$ often occurs in human hepatocellular carcinoma (HCC). Point mutations that affect phosphorylation sites or amino acid residues in CTNNB1 exon-3 have been reported in a large number ( $8 \%$ to $44 \%$ ) of HCCs. ${ }^{14,20-29}$

1,4-Bis[2-(3,5-dichloropyridyloxy)]benzene (TCP), the most potent agonist of constitutive androstane receptor $(\mathrm{CAR}),{ }^{30}$ is a powerful inducer of hepatocyte prolifera$\operatorname{tion}^{31,32}$ and a nongenotoxic mouse hepatocarcinogen ${ }^{33-35}$; in diethylnitrosamine (DENA)-pretreated mice it acts as a powerful liver tumor promoter. ${ }^{31,36,37}$

Experimental models of chemically induced hepatocarcinogenesis have confirmed the high frequency of $\beta$-catenin mutations observed in human HCC. Indeed, it has been shown that HCCs developed in mice initiated by DENA and promoted by chronic exposure to phenobarbital (PB), a powerful CAR activator, displayed a high frequency of $\beta$-catenin-activating mutations. Interestingly, the HCCs developed by DENA in the absence of CAR agonists showed mutations of Ha-ras, but not of $\beta$-catenin. ${ }^{38}$ From these results, it was proposed that the promoting activity of CAR agonists relies on their ability to confer a selective advantage to cells that carry DENA-induced $\beta$-catenin mutations.

However, this hypothesis cannot explain the complete tumorigenic activity exerted by TCP in the absence of genotoxic treatment. ${ }^{33-35}$

Because no information is available about the molecular alterations that lead to HCC development after chronic exposure to TCP alone, we investigated the possibility that CAR activation is per se sufficient to cause mutations and, consequently, HCC development.

\section{Materials and Methods}

\section{Animals and Treatment}

Six- to 8-week-old female $\mathrm{C} 3 \mathrm{H}$ mice were obtained from Charles River, Milan, Italy. Guide for the Care and Use of Laboratory Animals ${ }^{39}$ were followed throughout the investigation. All animal procedures were approved by the Ethical Commission of the University of Cagliari and the Italian Ministry of Health.

\section{Experimental Protocol 1}

Mice were injected intraperitoneally with DENA at a dose of $90 \mathrm{mg} / \mathrm{kg}$ body weight. After a 1-week recovery period, mice were treated intragastrically with TCP, $(3 \mathrm{mg} / \mathrm{kg}$ body weight; Sigma-Aldrich, St. Louis, MO) once weekly for 21 or 28 weeks. Another group was given only TCP once weekly for 28 and 42 weeks. Age-matched mice treated with dimethyl sulfoxide dissolved in corn oil were used as controls. The animals were euthanized at 21, 28, and 42 weeks, respectively; time points when HCCs were observed in both regimen protocols.

\section{Experimental Protocol 2}

Mice were treated with TCP $(3 \mathrm{mg} / \mathrm{kg}$ body weight $)$ once weekly for $1,12,16$, and 21 week.

\section{Laser-Capture Microdissection}

Sixteen preneoplastic lesions were microdissected from four DENA + TCP mice sacrificed at 21 weeks and random areas from the liver of TCP alone and age-matched control mice, as described previously. ${ }^{40}$ RNA was extracted from microdissected samples by using the mirVana miRNA Isolation Kit (Thermo Fisher Scientific, Waltham, MA), according to the manufacturer instructions.

\section{Quantitative RT-PCR Analysis}

Total RNA was extracted from frozen liver samples by using Trizol Reagent (Thermo Fisher Scientific), according to the manufacturer's protocol. Total and microdissected RNA was retro-transcribed by using the High Capacity cDNA Reverse Transcription Kit (Thermo Fisher Scientific). Analysis of Glul, Lgr5, Rgn, Lect2, Ccnd1, Tbx3, Axin2, and Ctnnb1 was performed by using specific TaqMan probes (Thermo Fisher Scientific) and Gapdh as the reference gene.

\section{Sanger Sequencing}

A mutation analysis was performed on the same cDNA samples used for quantitative RT-PCR (RT-qPCR) analysis. Primers were designed to PCR-amplify the region spanning exons 2 and 3 of Ctnnbl (forward: 5'-GGCCGCGAGTGAGCAG- $3^{\prime}$ ), annealing inside exon 1 (reverse: $5^{\prime}$-TTAGTGGGATGAGCAGCGTC- ${ }^{\prime}$ ), annealing inside exon 4 (annealing temperature $60^{\circ} \mathrm{C}$ ). For $\mathrm{Ha}$-ras, the region spanning exons 2 and 3 (codons 13 to 97) (forward: 5'-AGTCGCGCCAGCAAGC-3'), annealing inside exon 1 (reverse: 5' AGACTCAACAGTGCGAGCAG-3'), annealing inside exon 4 (annealing temperature $60^{\circ} \mathrm{C}$ ) were PCR-amplified. All PCR products were amplified with High-Fidelity Taq polymerase (Platinum Taq DNA Polymerase High Fidelity; Thermo Fisher Scientific), purified (by exonuclease 1 and shrimp alkaline phosphatase; GE Healthcare Life Sciences, Pittsburgh, PA), and sequenced by fluorescent-based Sanger's direct sequencing in an ABI 3130 DNA capillary sequencer. The mutation nomenclature described in this study is given according to the numbering of the amino acids in the human protein sequence (Ensembl Release 90). 


\section{mRNA Expression Profiling}

RNA was extracted by using the miRNeasy Mini Kit (Qiagen, Hilden, Germany) from three control mice, three TCP mice, nine HCCs (DENA + TCP), and seven HCCs (TCP). For the gene expression profile, $150 \mathrm{ng}$ of RNA was amplified (TotalPrep RNA Amplification Kit; Illumina Inc., San Diego, CA), labeled, and hybridized on Illumina microarray (MouseWG-6-V2_0 BeadChips; Illumina Inc.), including 21,791 genes.

\section{Microarray Data Analysis}

The intensity files were loaded into the Illumina BeadStudio software version 3.0.19.0 (Illumina Inc.) and BRB Array Tools version 4.2.0 for quality control and gene expression analysis. First, the quantile normalization algorithm was applied on the data set. Only genes whose expression differed by at least 1.5 -fold from the median in at least $20 \%$ of the arrays and characterized by a 50th percentile of intensities $>300$ were retained. The false discovery rate-adjusted $P$ values were calculated by using the Benjamini-Hochberg procedure. ${ }^{41}$ According to these criteria, 2281 expressed transcripts of 21,791 showed reproducible up- or down-regulation. To identify the differentially expressed genes, Random-Variance Model and Multivariate Permutation Test were applied. After this analysis, 1784 genes showed reproducible up- or downregulation in at least one comparison (gene array data are available at Gene Expression Omnibus, https://www.ncbi. nlm.nih.gov/geo; accession number GSE113708).

Mouse standard gene symbols were submitted to the Ingenuity pathway analysis (IPA) pipeline. Analysis of Pathways and Function was based on the number of genes significantly dysregulated (fold difference cutoff \pm 2.0 ). mRNA validation was performed by using specific TaqMan assays (Thermo Fisher Scientific) for the following genes, Cyp2c39, G6pc, Diol, and Sp5, using Gapdh as reference gene.

\section{Immunohistochemistry}

Formalin-fixed, paraffin-embedded sections were incubated overnight with the following antibodies: anti-GS (SigmaAldrich) and anti- $\beta$-catenin (Santa Cruz Biotechnology, Dallas, TX) and were detected by anti-rabbit horseradish peroxidase antibody (Dako Envision, Santa Clara, CA) and 3,3'-diaminobenzidine (Sigma-Aldrich). Sections were counterstained with Harris hematoxylin.

\section{Protein Extraction and Western Blot Analysis}

Mouse liver samples were lyzed with the tissue protein extraction reagent (Thermo Fisher Scientific). Western blot analyses were performed according to standard methods. The following antibodies were used: anti-GS
(Sigma-Aldrich), anti-nonphospho (Active) $\beta$-Catenin (S33/37/T41) (Cell Signaling, Danvers, MA), anti-GPCR49 and anti-Cyclin D1 (Abcam, Cambridge, UK), antiRegucalcin (Santa Cruz Biotechnology), anti- $\beta$-catenin (Becton Dickinson, Franklin Lakes, NJ), and anti- $\alpha$-tubulin (Cell Signaling). The enhanced chemiluminescence system (Thermo Fisher Scientific) was used for the final detection.

\section{Statistical Analysis}

Analysis of significance was performed by $t$-test, with Welch's correction when variances were unequal or by oneway analysis of variance with the use of the Instat GraphPad Prism 5 (GraphPad Software Inc., San Diego, CA). The results of observations are presented as the means \pm SEM. A value of $P<0.05$ was considered as a significant difference between groups.

\section{Results}

Ctnnb1 Mutations Are Highly Frequent in HCCs Developed by DENA, followed by Chronic Exposure to TCP

Treatment with the CAR agonist PB after DENA treatment promoted the growth of HCCs characterized by a high frequency of $\beta$-catenin mutations. ${ }^{38}$ TCP was also a powerful CAR agonist and a liver tumor promoter ${ }^{31,34-37}$; however, whether the molecular alterations were similar to those caused by PB was unknown. The exon 3 of Ctnnbl was analyzed by using Sanger fluorescence-based sequence analysis, in neoplastic lesions arising at 21 and 28 weeks after a single DENA administration, followed by weekly doses of TCP.

First, 16 neoplastic lesions identified by hematoxylin and eosin staining as low-grade and high-grade dysplastic nodules were laser-microdissected 21 week after DENA. These nodules displayed a heterogeneous cellstructure, with a predominance of large, polygonal ground-glass-like hepatocytes that showed cellular atypia, which was more evident in high-grade dysplastic nodules.

Ctnnb1 mutations were found in 13 of 16 neoplastic lesions $(81 \%)$ and involved mainly the recognition site for the $\beta$-transducing repeat-containing protein ubiquitin ligase ( $n=9$ of 13), with substitutions at D32 (to G), S33 (to A), I35 (to S), and S37 (to F or to P). Three of 13 point mutations were found in the degradation box of $\beta$-catenin at residue 41 (T41A), whereas only one sample showed large in-frame deletion or exon skipping. Notably, four mutations were the same as those found when PB was used as a promoting agent $^{38}$ (Supplemental Table S1). No tumors were observed in the liver of age-matched controls or mice treated with TCP alone. These data suggested that, in this model, Ctnnbl mutations preceded the development of frankly malignant HCCs. 
In humans, CTNNB1 mutations affect exon 3 at specific sites involved in the degradation of $\beta$-catenin protein, leading to its stabilization, nuclear translocation, and transcriptional activation of its target genes. ${ }^{5-7}$ To assess whether DENA + TCP-induced Ctnnb1 mutations resulted in increased $\beta$-catenin transcriptional activity, the expression levels of two well-known $\beta$-catenin target genes, Glul and Lgr5, were analyzed by RT-qPCR in the same microdissected tumors used for mutation analysis. The results showed that Glul and $\operatorname{Lgr} 5$ were up-regulated in most of the neoplastic lesions compared with aged-matched control and TCP-alone mice (Supplemental Figure S1). Up-regulation of Glul and Lgr5 was highly significant in $\beta$-catenin-mutated samples compared with nonmutated tumors $(P<0.0001$ and $P<0.01$, respectively). Unexpectedly, comparison of the mRNA levels of the same genes between TCP-treated and control livers indicated that repeated treatment with TCP caused either down-regulation or no change.

Twenty-eight weeks after DENA + TCP, all animals developed HCC (Figure 1A), with Ctnnbl somatic mutations identified in most cancers $(n=17$ of $20,85 \%)$ (Table 1). Point mutations in the recognition site for the $\beta$-transducing repeat-containing protein ubiquitin ligase were particularly frequent ( $n=13$ of 17$)$, with substitutions at D32 (to G), S33 (to A or P), I35 (to S), and S37 (to P); 2 of 17 mutations were found in the degradation box of $\beta$-catenin at residue T41 (to A), whereas two HCCs showed double mutations (S33P and I35S). At this stage, six mutations that are not present in the 21-week tumors that involved codon 33 (TCT-CCT) and have already been described in the DENA + PB model were found. ${ }^{38}$

The expression levels of Glul and Lgr5 were higher in most of the HCCs compared with those of control samples
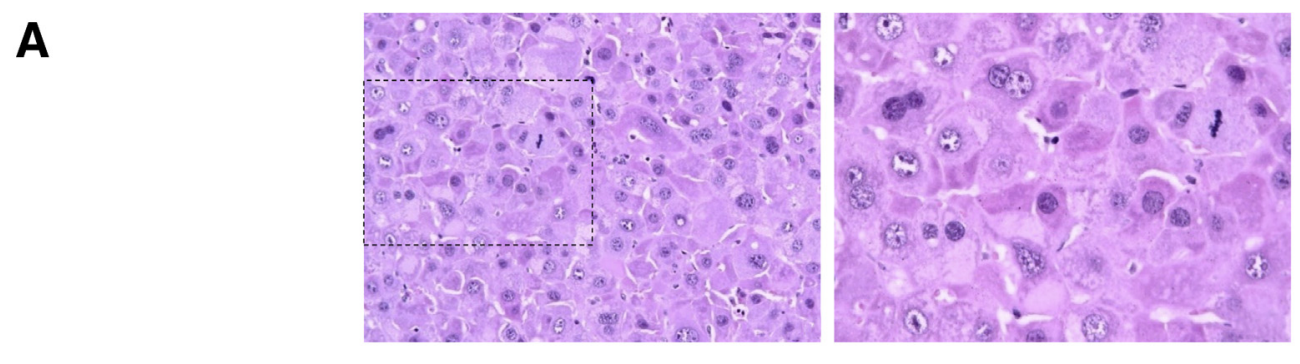
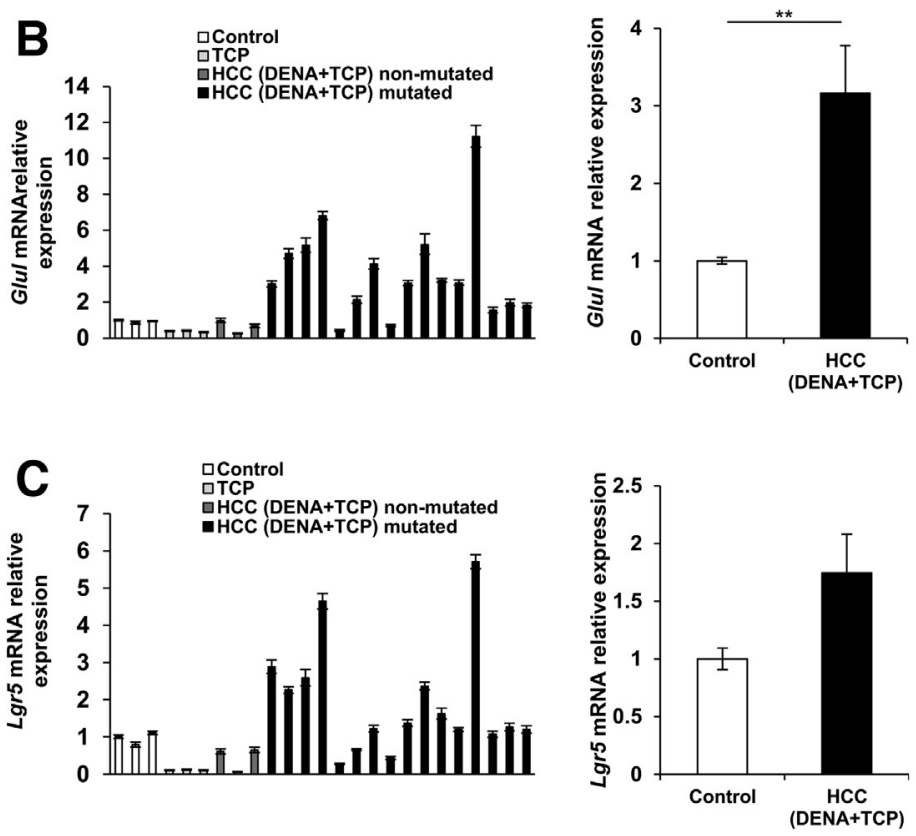
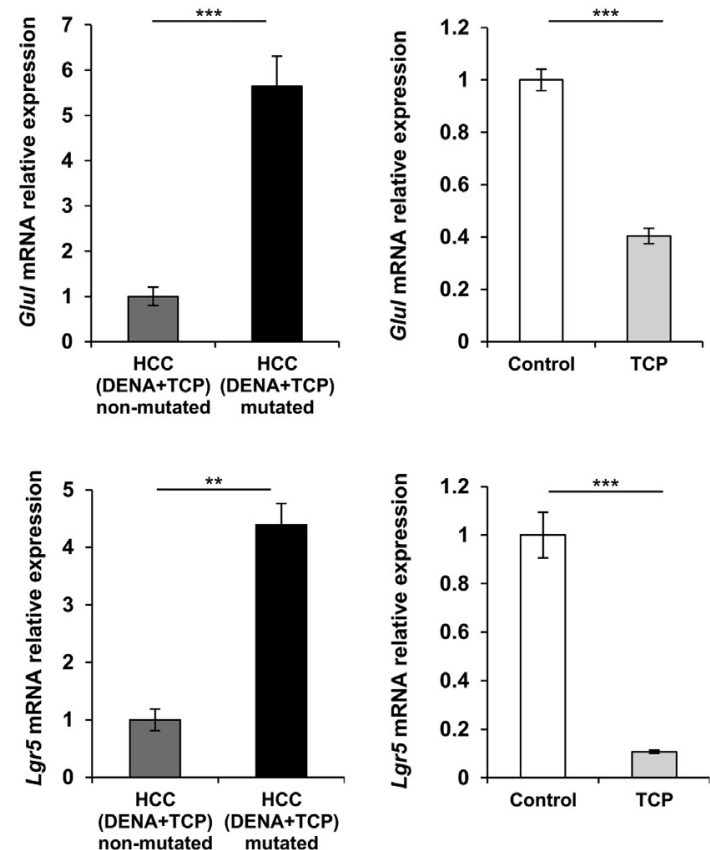

Figure 1 Gene expression of $\beta$-catenin target genes in DENA + 1,4-bis[2-(3,5-dichloropyridyloxy)]benzene (TCP)-treated mice (28 weeks). A: Representative microphotograph of a hepatocellular carcinoma (HCC) displaying cellular and nuclear atypia, mitotic figures, and lack of trabecular architecture (hematoxylin and eosin staining). Boxed area is shown at higher magnification in the right panel. B and C: Quantitative RT-PCR analysis of Glul (B) and Lgr5 (C). mRNA levels in controls, TCP, nonmutated HCCs, and mutated HCCs. The levels of both target genes were reported as relative mRNA expression by using the $2^{-\Delta \Delta C t}$ method and mouse Gapdh as reference gene (left panel). The middle panel and the right panel represent the average levels of Glul and Lgr5, respectively, calculated as fold change difference between nonmutated versus mutated HCCs, and controls versus TCP, respectively. Error bars represent SEM. $n=3$ control mice; $n=3$ TCP-treated mice; $n=3$ nonmutated HCC samples; $n=16$ mutated HCC samples. ${ }^{* *} P<0.01$ and ${ }^{* * * P} P 0.001$ ( $t$-test). Original magnification: $\times 20($ A, left panel); $\times 40($ A, right panel). 
Table 1 Ctnnb1 Mutations in DENA + TCP-induced HCCs (28 Weeks)

\begin{tabular}{|c|c|c|c|}
\hline Group & $\begin{array}{l}\text { Nucleotide } \\
\text { substitution }\end{array}$ & $\begin{array}{l}\text { Amino acid } \\
\text { substitution }\end{array}$ & $\begin{array}{l}\text { Mutated samples, } \\
n(\text { total } \\
\text { samples }=20)\end{array}$ \\
\hline HCC (DENA + TCP) & $\mathrm{TCT}>\mathrm{CCT}$ & S33P*† & 6 \\
\hline \multirow[t]{5}{*}{28 weeks } & $\mathrm{ATC}>\mathrm{AGC}$ & I35S* & 6 \\
\hline & $\mathrm{GAT}>\mathrm{GGT}^{\dagger}$ & $\mathrm{D} 32 \mathrm{G}^{\dagger}$ & 2 \\
\hline & $\mathrm{TCT}>\mathrm{CCT}^{\dagger}$ & $\mathrm{S} 37 \mathrm{P}^{\dagger}$ & 2 \\
\hline & $\mathrm{ACC}>\mathrm{GCC}^{\dagger}$ & $\mathrm{T}_{41 \mathrm{~A}^{\dagger}}$ & 2 \\
\hline & $\mathrm{TCT}>\mathrm{GCT}^{\dagger}$ & $\mathrm{S} 33 \mathrm{~A}^{\dagger}$ & 1 \\
\hline
\end{tabular}

Sanger analysis was performed in $20 \mathrm{HCC}$ from eight mice treated with a weekly dose of TCP for 28 weeks after a single dose of DENA.

*These two mutations were identified in the same lesion in two different samples.

${ }^{\dagger}$ Mutations that have been already described. ${ }^{38}$

DENA, diethylnitrosamine; HCC, hepatocellular carcinoma; TCP, 1,4-bis [2-(3,5-dichloropyridyloxy)] benzene.

(Figure 1, B and C). Notably, mRNA levels of Glul and $L g r 5$ were significantly higher in Ctnnbl-mutated samples than the nonmutated ones $(P<0.001$ for $G l u l$ and $P<0.01$ for $L g r 5)$. Similar to what was observed at 21 weeks, a significant down-regulation of Glul and Lgr5 was found in the liver of mice treated with TCP alone compared with that of age-matched control mice $(P<0.001)$.

These results suggested that sustained activation of CAR by repeated treatment with TCP led to a decrease of the $\mathrm{Wnt} / \beta$-catenin signaling pathway in nontumoral liver, whereas cancer cells that carried Ctnnbl mutations were able to escape the inhibitory effect exerted by CAR on this pathway.

\section{CAR Activation per se Is Sufficient to Cause Ctnnb1 Mutations}

Long-term exposure to TCP not only promoted HCC development after initiation by carcinogen administration but also induced the occurrence of HCC in the absence of genotoxic pretreatment. ${ }^{33-35}$ Although its promoting activity has been explained on the basis of the capacity of TCP to select carcinogen-initiated cells that harbor activating mutations in the $\beta$-catenin gene, ${ }^{38}$ the molecular alterations responsible for HCC development in the absence of genotoxic administration were totally unknown. To address this question, Ctnnbl mutations were examined in HCCs that developed 42 weeks after TCP treatment alone (Figure 2A). Under these experimental conditions, HCCs were observed in $100 \%$ of mice ( $n=7$ of 7 ). Treatment with TCP alone induced multiple lesions with more than a fourfold increase in liver size, compared with that of vehicle-treated mice $(1.31 \pm 0.003$ versus $5.75 \pm 0.072)$ (Figure 2B). Histologic analysis of tumors showed an altered structure of hepatocytes with nuclear atypia, pleomorphism, and frequent mitoses (Figure 2C). Notably, CAR activation of HCC cells compared with control livers was demonstrated by the highly significant up-regulation of two classical CAR target genes, Cyp2b10 and Gadd45b $b^{42,43}$ (Supplemental Figure S2).

HCCs were characterized by a high frequency of Ctnnbl somatic mutations $(n=10$ of $11 ; 90.9 \%)$. Mutations
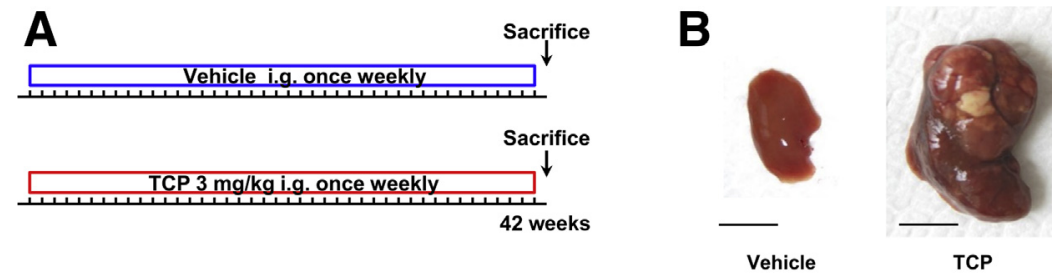

TCP
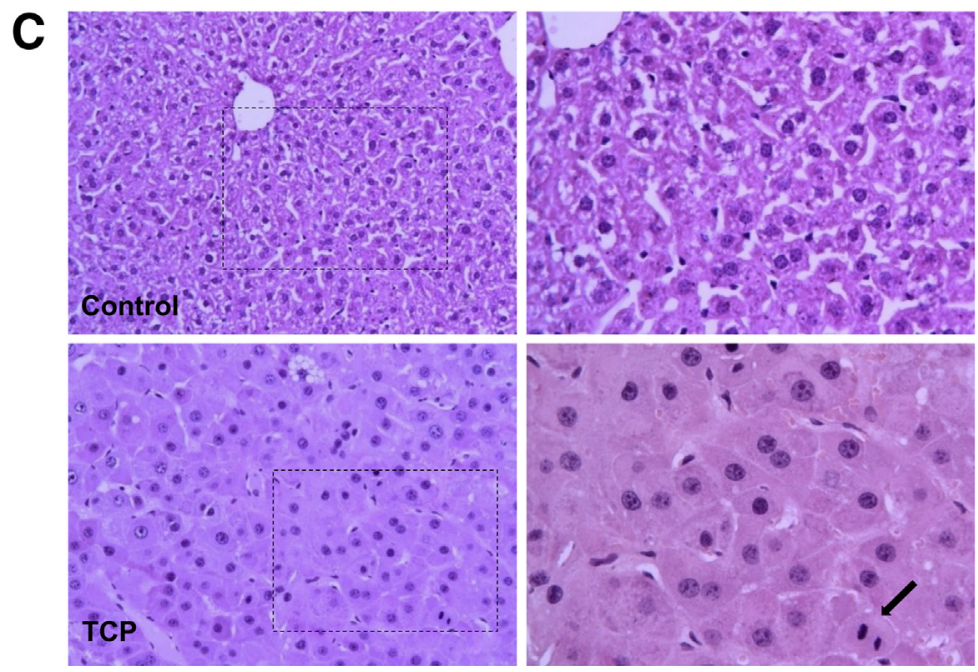

Figure 2 Repeated treatment with 1,4-bis[2(3,5-dichloropyridyloxy)]benzene (TCP) alone induced hepatocellular carcinoma (HCC) development. A: Experimental protocol: mice were treated intragastrically with corn oil or TCP ( $3 \mathrm{mg} / \mathrm{kg}$ body weight) once weekly for 42 weeks. B: Enlarged liver displaying several macroscopic tumors were evident in mice treated with vehicle or TCP for 42 weeks. C: Representative microphotograph showing the liver of mice treated with vehicle or TCP for 42 weeks. Neoplastic cells arranged in thick layers and displaying increased nuclear/cytoplasmic ratio and pleomorphism were observed. Arrow indicates mitosis (hematoxylin and eosin staining). Boxed areas are seen at higher magnification in the right column. Scale bars $=1 \mathrm{~cm}$. Original magnification: $\times 20$ (C, left column); $\times 40$ (C, right column). 
resulted in 13 different nucleotide substitutions (Table 2); 6 of 10 mutations occurred at codons 32, 33, and 34 (D32N, S33Y, and G34 to E or R), with a different pattern of nucleotide substitutions compared with the DENA + TCP group. In the remaining $40 \%$ of samples, in-frame deletions or exon skipping that targeted Ctnnb1 exon 3 (A5-A80 del, M14-G38 del) that prevented $\beta$-catenin degradation were identified. Notably, almost all these mutations, including large in-frame deletions (delA5-A80), have been reported in the $\beta$-catenin gene, in c-myc or Ha-ras transgenic mice, and in human HCCs. ${ }^{20,29,44}$

Because these mutations, although putative-activating mutations, were distinct from those found in DENA + TCP HCCs, their effect on the induction of $\beta$ catenin target genes was determined. Unexpectedly, the results showed that, differently from HCCs of mice treated with DENA + TCP, the mutations found in HCCs from mice treated with TCP alone did not affect the expression of Glul and Lgr5 compared with the corresponding agematched mice treated with the vehicle (Figure 3, A and B). Moreover, when the mRNA levels of five additional $\beta$-catenin target genes were examined, either no change (Lect-2, Axin2, and $T b x 3)$ or down-regulation (Ccndl and Rgn) was observed (Figure 3, C-G). With the only exception of Cyclin D1, Western blot analysis showed no difference or a decrease in the protein content of Regucalcin, GS, and $\operatorname{Lgr} 5$, thus confirming the results obtained by RT-qPCR (Supplemental Figure S3). Altogether, the results showed that $\beta$-catenin mutations found in $\mathrm{HCCs}$ generated by TCP alone, unlike those observed in HCCs from DENA-TCP-treated mice, did not induce the transcription of classic $\beta$-catenin target genes.

Ha-ras mutations were previously found in animals treated with DENA alone, but they were not found in mice injected with DENA and then exposed to PB administration. ${ }^{38}$ To establish whether Ha-ras mutations could occur after TCP treatment, HCCs from both DENA + TCP (28 weeks) and TCP alone (42 weeks) mice were also screened for Ha-ras mutations. However, no mutation was detected in any of the 31 tumors analyzed.

Table 2 Ctnnb1 Mutations in HCCs Induced by TCP Alone (42 Weeks)

\begin{tabular}{llll}
\hline Group & $\begin{array}{l}\text { Nucleotide } \\
\text { substitution }\end{array}$ & $\begin{array}{l}\text { Amino acid } \\
\text { substitution }\end{array}$ & $\begin{array}{l}\text { Mutated samples, } n \\
\text { (total samples }=11 \text { ) }\end{array}$ \\
\hline HCC (TCP) & GCT-GCT & A5-A80 del & 3 \\
42 weeks & GGA > GAA & G34E & 3 \\
& GGA $>$ AGA & G34R & 1 \\
& ATG-GGT & M14-G38 del & 1 \\
& GAT>AAT & D32N & 1 \\
& TCT>TAT & S33Y & 1 \\
\hline
\end{tabular}

Sanger analysis was performed in $11 \mathrm{HCC}$ from five mice treated with a weekly dose of TCP for 42 weeks.

HCC, hepatocellular carcinoma; TCP, 1,4-bis[2-(3,5-dichloropyridyloxy)] benzene.
Transcriptomic Analysis Reveals HCCs Cluster in Two Subclasses

Previous work has shown that gene expression patterns of mouse HCCs generated by the genotoxic carcinogen DENA are quite similar to human HCCs with the poorer survival, ${ }^{45}$ whereas those of HCCs from mice treated with the nongenotoxic ciprofibrate, a potent peroxisome proliferator and a peroxisome proliferator-activated receptor $\alpha$ ligand, ${ }^{46}$ were not. To explore whether a similar difference in the pattern of gene expression could take place in HCCs induced by genotoxic versus nongenotoxic conditions, hierarchical clustering analysis of gene expression patterns was applied to assess the relative similarities between HCCs generated by DENA + TCP and those induced by TCP alone. Hierarchical cluster analysis of 2281 genes stratified the liver samples into two major clusters: i) control livers and TCP at 28 weeks and ii) HCCs generated by DENA + TCP and by TCP alone (Figure 3H). Notably, the only HCC that grouped together with TCP alone and controls was the only one devoid of Ctnnbl mutations. As shown by the Heat Map, although HCCs from DENA + TCP and those from TCP-alone treated mice could not be clearly subgrouped, controls were distinctly separated by nontumoral livers of mice given TCP alone for 28 weeks. RT-qPCR validation performed on randomly selected genes (Cyp2c39, Dio1, G6pc, Sp5) confirmed the microarray expression data for all four examined genes (Supplemental Figure S4).

In accordance with the heat map, IPA of genes altered in HCCs did not reveal major differences between HCCs generated with or without DENA (Supplemental Figure S5A). Indeed, not more than four genes for each pathway were differentially expressed for a twofold or greater difference between the two groups, with most of the dysregulated genes being involved in metabolic pathways. Functional investigation also underlined a common pattern and identified lipid metabolism, molecular transport, and small molecule biochemistry as the most significantly affected (Supplemental Figure S5B). However, when IPA was applied to investigate the transcription factor-dependent pathways, the results showed that the one mediated by $\beta$-catenin was the most differentially modified between DENA-TCP HCC and TCP HCC (Supplemental Figure S6).

When IPA was applied to control samples and TCP nontumoral livers, major pathway modifications were found, as indicated by a much higher number of differentially expressed genes in the two groups (Supplemental Figure S5A). Among the pathways, alterations in nuclear receptor-dependent pathways were particularly frequent [pregnane X receptor (XR)/retinoid XR activation, lipopolysaccharide/IL-1-mediated inhibition of retinoid XR function, farnesoid XR/retinoid XR activation, and liver $\mathrm{XR} /$ retinoid XR activation]. Similar to the analysis performed on HCCs, functional investigation showed lipid 
A

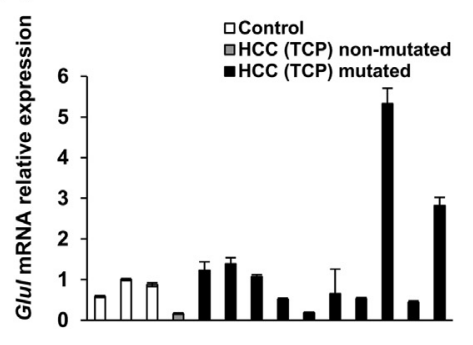

C

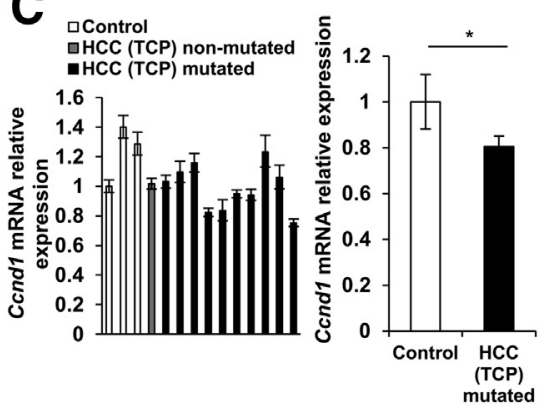

$\mathbf{F}$

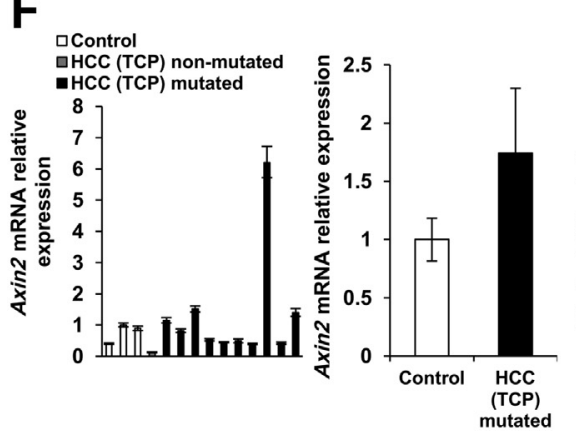

B

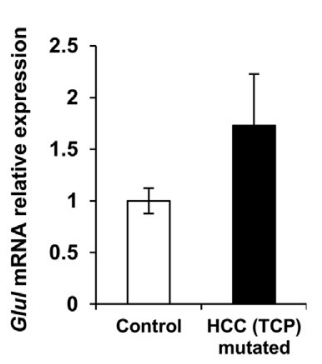

D
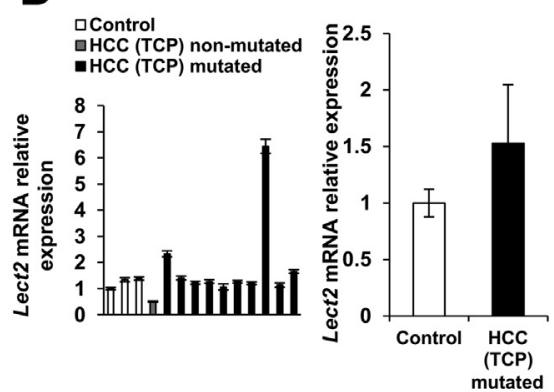

$E_{\text {acontrol }}$

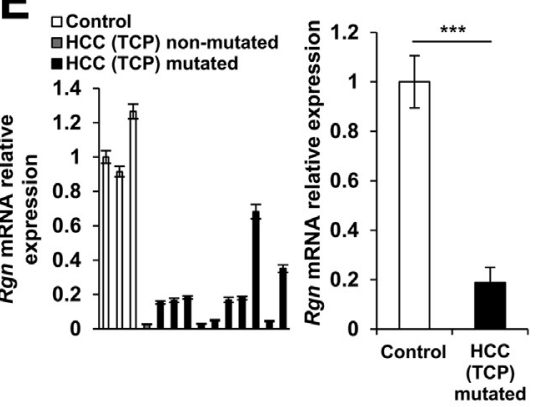

G

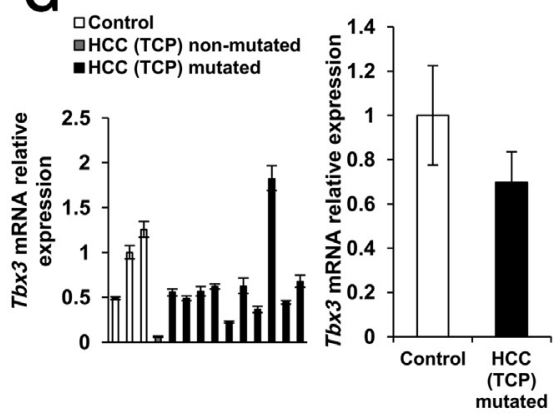

H

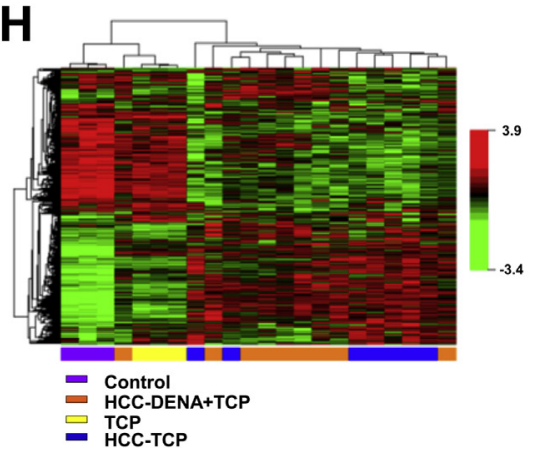

Figure 3 mRNA levels of $\beta$-catenin target genes and transcriptomic analysis of hepatocellular carcinomas (HCCs). A-G: Quantitative RT-PCR analysis of Glul (A) Lgr5 (B), Cond1 (C), Lect2 (D), Rgn (E), Axin2 (F), and Tbx3 (G) mRNA levels in controls and HCCs from mice treated with 1,4-bis[2-(3,5dichloropyridyloxy)]benzene (TCP) for 42 weeks. The levels of both target genes are reported as relative mRNA expression by using the $2^{-\Delta \Delta C t}$ method and mouse Gapdh as reference gene. The average levels of all genes analyzed were calculated as fold-change difference between $\mathbf{H}$ : Hierarchical clustering of 2281 genes in normal liver (control), liver from mice treated with TCP alone for 28 weeks, and HCCs developed in mice treated with diethylnitrosamine (DENA) + TCP (HCC-DENA + TCP) or TCP alone (HCC-TCP). Each row represents the expression profile of a gene. Columns represent single samples. Red and green colors represent higher or lower (3.9 and -3.4) expression levels of the mRNA (median-centered), respectively. Error bars represent SEM. $n=3$ control samples $(\mathbf{A}-\mathbf{G}) ; n=11 \mathrm{HCC}$ samples from mice treated with TCP for 42 weeks $(\mathbf{A}-\mathbf{G}) ; n=10$ TCP-mutated HCC samples $(\mathbf{A}-\mathbf{G})$. ${ }^{*} P<0.05$ and $* * * P<0.001$ (t-test).

metabolism, molecular transport, and small molecule biochemistry with the most modified functions (Supplemental Figure S5B).

\section{Inhibition of GS Protein Content after Chronic Exposure to TCP}

Concomitant activation of CAR and $\beta$-catenin induced uncontrolled liver growth and tumorigenesis. ${ }^{47}$ However, the results showed that CAR activation by TCP lead to down-regulation of $\beta$-catenin target genes in nontumoral liver. To further characterize the effect of TCP on the Wnt/ $\beta$-catenin pathway, the levels of the GS protein were evaluated in liver samples 28 and 42 weeks after the beginning of the experiment. In agreement with mRNA levels, Western blot analysis in total tissue lysates revealed a strong decrease of GS in mice treated with TCP alone, compared with age-matched controls or HCCs generated by treatment with DENA + TCP (Figure 4A). This decrease was not due to reduced expression of $\beta$-catenin, because no change in mRNA or protein levels of $\beta$-catenin (total and active) was found between control mice and TCP-treated mice (Supplemental Figure S7 and Figure 4A).

In addition, immunostaining revealed that, although perivenular hepatocytes were strongly positive for GS in control livers at 28 or 42 weeks, an almost complete loss of 
A

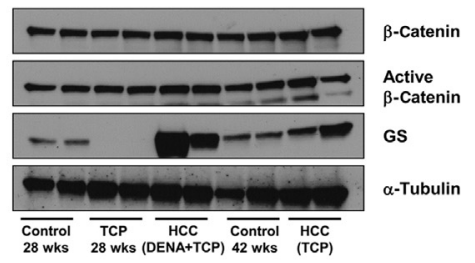

B
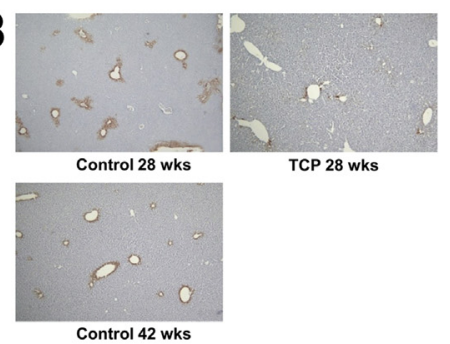

C

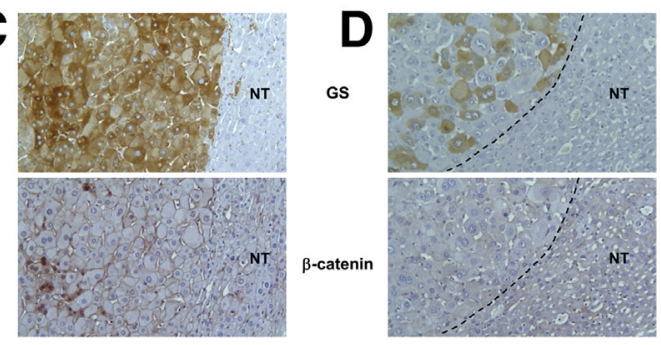

E
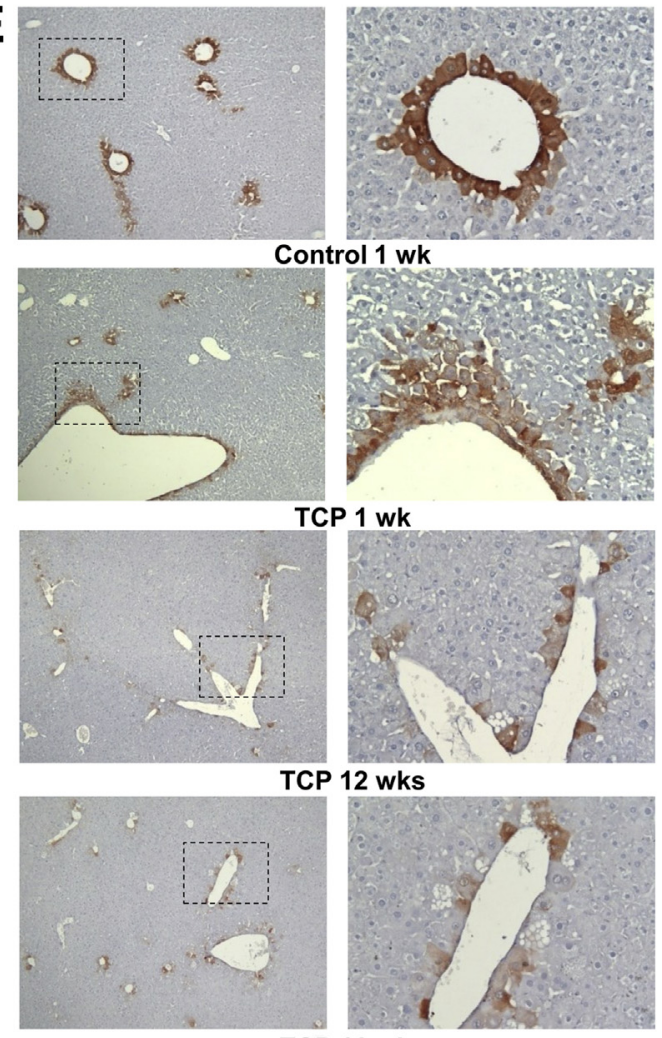

TCP 16 wks

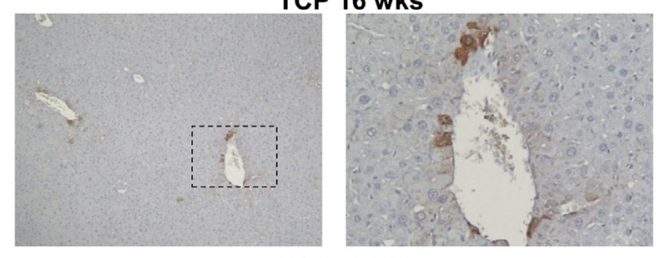

TCP 21 wks the protein was observed in TCP-treated mice after 28 weeks of treatment (Figure 4B). In agreement with RTqPCR analysis, HCCs developed in DENA-pretreated mice displayed an intense GS staining that was virtually absent in the peritumoral regions (Figure 4C). Serial sections showed several hepatocyte nuclei strongly positive for $\beta$-catenin, further supporting the activating nature of the mutations found in HCCs from DENA + TCP mice (Figure 4C). Staining of GS was observed, albeit at a lower degree, also in the tumors generated by 42 weeks of treatment with TCP alone (Figure 4D). However, no nuclei positive for $\beta$-catenin were virtually observed in these tumors (Figure 4D).

To assess whether inhibition of the Wnt/ $\beta$-catenin pathway occurred as a consequence of acute or chronic TCP exposure, additional experiments were performed whereby mice were sacrificed at $1,12,16$, and 21 weeks after treatment with TCP (Supplemental Figure S8A). TCP treatment induced a strong increase in the liver/body weight ratio at all time points examined (Supplemental Figure S8B). Microscopic analysis of the TCP-induced enlarged livers did not reveal significant pre- or neoplastic changes, although dysplastic hepatocytes with increased nuclear/cytoplasmic ratio were frequently observed (data not shown). A single treatment with TCP did not modify the number of GS-positive cells surrounding the perivenous region, compared with control livers. However, a clear loss of staining was found after 12 weeks of weekly TCP treatment, which persisted along the other time points (Figure 4E).

\section{Discussion}

The main and novel finding of this work is that the tumorigenic property of the CAR ligand, TCP, is associated with an extremely high frequency of $\beta$-catenin mutations, independent of a genotoxic agent preadministration. Two

Figure 4 Down-regulation of glutamine synthase (GS) in nontumoral livers of 1,4-bis[2-(3,5-dichloropyridyloxy)] benzene (TCP)-treated mice. A: Western blot analysis of total $\beta$-catenin, active $\beta$-catenin, and GS from livers of controls, TCP, and hepatocellular carcinomas (HCCs) induced by diethylnitrosamine (DENA) + TCP at 28 weeks, and controls and HCCs induced by TCP alone at 42 weeks. Each lane represents an individual sample. $\alpha$-Tubulin was used as loading control. B: Immunostaining for GS in controls (28 and 42 weeks) and TCP-treated mice (28 weeks). Note the almost complete absence of GS around the terminal hepatic veins in TCPtreated mice. C: Microphotograph showing a strong GS immunostaining in HCC from DENA + TCP at 28 weeks (top), and the presence of several $\beta$ catenin-positive nuclei in a serial section stained for $\beta$-catenin (bottom). D: GS (top) and $\beta$-catenin (bottom) immunostaining in HCCs from mice receiving only TCP for 42 weeks. GS was virtually absent in nontumoral (NT) liver; dashed lines separate HCC (left) from NT tissue (right). E: GS immunostaining in control and TCP-treated mouse liver 1 week after a single dose of TCP or vehicle and at 12, 16, and 21 week of administration of the constitutive androstane receptor agonist. Boxed areas are shown at higher magnification in the right column. Original magnification: $\times 5$ (B and $\mathbf{E}$, left column $) \times 20($ C, D, and $E$, right column $)$. 
possibilities can be envisaged to explain this result: the tumorigenic activity of TCP, unlike what is currently thought, does not rely on a selective process on hepatocytes that harbor carcinogen-induced $\beta$-catenin mutations, but rather on its ability to generate an environment that favors mutations of this gene in some hepatocytes (such as the hepatic inflammatory response observed after PB treatment) ${ }^{48}$; alternatively, spontaneously $\beta$-catenin-mutated hepatocytes are present in normal mouse liver which are then selected by CAR once it is activated by its ligands.

Although at present the reason for the high number of $\beta$-catenin mutations in the absence of genotoxic treatment remains elusive, it is remarkable that mutations of Ctnnb1 in HCCs generated by TCP alone are strikingly different from those of mice pretreated with DENA. Indeed, although located in exon 3, they displayed a different pattern of nucleotide substitutions compared with DENA + TCP animals. Moreover, in $40 \%$ of the samples, large in-frame deletions/exon skipping that target Ctnnb1 exon 3 (A5-A80 del, M14-G38 del) that prevent $\beta$-catenin degradation were observed in the former, but not in the latter group. In this context, note that large in-frame deletions, in particular delA5-A80, have been reported in c-myc or Ha-ras transgenic mice ${ }^{20}$ and in $10 \%$ of human borderline adenomas/HCC lesions. ${ }^{44}$ Although in humans they are considered mutations with high activity, in mouse HCCs they do not significantly affect the activity of $\beta$-catenin.

Overall, although Ctnnb1 mutations in DENA + TCP HCCs positively correlate with the expression of $\beta$-catenin target genes, Glul and Lgr5, this was not the case in HCCs from mice exposed to TCP alone. Furthermore, HCCs generated by TCP treatment in the absence of DENA also displayed down-regulation of $R g n$, an additional $\beta$-catenin target gene. This finding suggests that the $\beta$-catenin mutations observed in DENA-treated mice confer a stronger growth advantage to the hepatocytes that harbor them, thus resulting in an accelerated tumor formation (21 week) compared with those found in mice treated with TCP alone, in which HCCs are observed at a much later time point. It is possible that some of the mutations in Ctnnbl that occur in HCC in the TCP group alone may lead to a conformational change in $\beta$-catenin structure, disallowing formation of proper transcriptional complex with transcriptional factors and other components, such as histone acetyltransferases, leading to lack of expression of target genes. Subcloning some representative mutants of Ctnnbl from HCC after treatment with TCP alone and examining their effect compared with that of mutants from DENA + TCP will be required to directly address if quality of mutations may differentially regulate $\beta$-catenin-TCF activity.

Regardless of the reason for the lack of induction of target gene expression, note that a subset of HCC patients who did not exhibit up-regulation of $4 \beta$-catenin target genes ( $\mathrm{Glul}$, Axin2, Lect2, and Rgn), despite nuclear $\beta$-catenin translocation, showed the poorest survival. ${ }^{49}$ Thus, up-regulation of $\beta$-catenin target genes may not always predict its activation and/or mutation.

Of note, the different mutations found in the two groups do not appear to lead to any major modification of the gene expression profile of HCCs. Indeed, the molecular profiling of HCCs developed in DENA + TCP versus TCP did not identify any significant difference between HCCs generated by a genotoxic agent (DENA) and those caused by repeated administration of TCP alone. This is different from a previous observation showing that gene expression patterns of mouse HCCs induced by genotoxic agents were similar to those of the poorer survival group of human HCCs, whereas patterns of HCCs from mice treated with nongenotoxic ligands of peroxisome proliferator-activated receptor $\alpha$ were not. ${ }^{45}$ Overall, the microarray analysis suggests that the expression profile of tumors carrying $\beta$-catenin mutations with different transcriptional activity does not allow their stratification into two different groups.

Another novel and intriguing finding of this work is that CAR activation by TCP, apart from being associated with the presence of Ctnnbl mutations in neoplastic hepatocytes, concomitantly exerts an inhibitory effect on the Wnt/B-catenin pathway in nontumoral liver. Indeed, a significant down-regulation of Glul and Lgr5 mRNA levels and a strong decrease of GS protein content, observed by both Western blot analysis and immunohistochemistry, was detected in the liver of TCP-treated mice, before tumor development. This suggests that only those hepatocytes carrying either spontaneous or CAR-induced Ctnnbl mutations can escape the suppressive effect of TCP on the Wnt/ $\beta$-catenin pathway and may clonally expand to develop HCC.

Down-regulation of the Wnt/ $\beta$-catenin pathway observed in the present study after treatment with the CAR agonist TCP is intriguing. Indeed, evidence of a functional interaction between CAR and $\beta$-catenin comes from the finding that most of the liver tumors generated by treatment with DENA followed by the promoting agent $\mathrm{PB}$, a CAR activator, display activating mutations in $\beta$-catenin, ${ }^{38,50}$ whereas no mutations are observed in tumors induced by DENA alone. Moreover, PB treatment is selective for a $\beta$-catenin-positive cell population in the c-Myc/transforming growth factor- $\alpha$ transgenic mouse model of HCC, and the activation of $\beta$-catenin provides proliferative and invasive advantages in this model. ${ }^{51}$ Furthermore, some works have shown that PB does not promote tumor development in liver-specific Ctnnbl knockout mice ${ }^{52}$ and that concomitant activation of CAR and $\beta$-catenin induces uncontrolled liver growth and tumorigenesis. ${ }^{47}$ Although these findings strongly suggest a cooperation between CAR and $\beta$-catenin, no clear evidence for alterations in transactivation or expression levels of $\beta$-catenin on CAR or vice versa has been observed. ${ }^{47}$ In the present study, long-term, but not short-term, CAR activation seems to negatively interfere with $\beta$-catenin activity as shown by decreased expression of the two $\beta$-catenin target genes examined, 
namely Glul and $L g r 5$, in the liver of mice treated with TCP for 28 weeks (Figure 1, B and C). This indicates that CAR can synergistically act on $\beta$-catenin only when the latter is mutated. The combinatory effect of CAR activation together with the occurrence of $\beta$-catenin mutations in few hepatocytes, in a context of a general impairment of the Wnt/ $\beta$ catenin pathway, may thus represent a selective advantage for $\beta$-catenin-mutated cells in their progression to HCC. The rapid availability of activated $\beta$-catenin after treatment with genotoxic agents (such as DENA) may explain the accelerated development of HCC compared with the group treated with TCP alone, in which a longer time is most likely required for the occurrence of gene mutation.

Some studies are beginning to report heterogeneity in Wnt signaling due to not only mutations in various components of the pathway such as AXIN and CTNNB $1^{29}$ but also within mutations that affect the same gene such as CTNNB1. ${ }^{44,49}$ Likewise, it is well known that $\beta$-catenin can be activated without CTNNB1 mutations, for example by transforming growth factor- $\beta$, and molecular classification of HCC in patients has identified different classes of patients owing to this heterogeneity. Future studies are needed to establish whether the observations stemming from our work might relate to human pathogenesis.

\section{Conclusion}

The present study identified in a nongenotoxic mouse tumor model the presence of $\beta$-catenin mutations in approximately $90 \%$ of the HCCs caused by chronic CAR activation. Because $\beta$-catenin mutations are among the most common mutations in human HCC, this mouse model, which is devoid of mutagenic/genotoxic agents, may provide a useful tool for a better understanding of the molecular events leading to the high frequency of $\beta$-catenin in human HCC, in particular of the possible role played by CAR activation.

\section{Supplemental Data}

Supplemental material for this article can be found at https://doi.org/10.1016/j.ajpath.2018.07.022.

\section{References}

1. Aberle H, Butz S, Stappert J, Weissig H, Kemler R, Hoschuetzky H: Assembly of the cadherin-catenin complex in vitro with recombinant proteins. J Cell Sci 1994, 107:3655-3663

2. Orsulic S, Huber O, Aberle H, Arnold S, Kemler: E-cadherin binding prevents beta-catenin nuclear localization and beta-catenin/LEF-1mediated transactivation. J Cell Sci 1999, 112:1237-1245

3. Lilien J, Balsamo J: The regulation of cadherin-mediated adhesion by tyrosine phosphorylation/dephosphorylation of beta-catenin. Curr Opin Cell Biol 2005, 17:459-465

4. Gumbiner BM: Regulation of cadherin-mediated adhesion in morphogenesis. Nat Rev Mol Cell Biol 2005, 6:622-634

5. Amit S, Hatzubai A, Birman Y, Andersen JS, Ben-Shushan E, Mann M, Ben-Neriah Y, Alkalay I: Axin-mediated CKI phosphorylation of beta-catenin at Ser 45: a molecular switch for the Wnt pathway. Genes Dev 2002, 16:1066-1076

6. Monga SP: Role and regulation of beta-catenin signaling during physiological liver growth. Gene Expr 2014, 16:51-62

7. Logan CY, Nusse R: The Wnt signaling pathway in development and disease. Annu Rev Cell Dev Biol 2004, 20:781-810

8. Cadigan KM, Nusse R: Wnt signaling: a common theme in animal development. Genes Dev 1997, 11:3286-3305

9. Peifer M, Polakis P: Wnt signaling in oncogenesis and embryogenesis - a look outside the nucleus. Science 2000, 287:1606-1609

10. Willert K, Brown JD, Danenberg E, Duncan AW, Weissman IL, Reya T, Yates JR III, Nusse R: Wnt proteins are lipid-modified and can act as stem cell growth factors. Nature 2003, 423:448-452

11. Nusse R: Wnt signaling in disease and in development. Cell Res 2005, 15:28-32

12. Polakis P: Wnt signaling in cancer. Cold Spring Harb Perspect Biol 2012, 4:a008052

13. Cadoret A, Ovejero C, Terris B, Souil E, Lévy L, Lamers WH, Kitajewski J, Kahn A, Perret C: New targets of beta-catenin signaling in the liver are involved in the glutamine metabolism. Oncogene 2002, 21:8293-8301

14. Yamamoto Y, Sakamoto M, Fujii G, Tsuiji H, Kenetaka K, Asaka M, Hirohashi S: Overexpression of orphan G-protein-coupled receptor, Gpr49, in human hepatocellular carcinomas with beta-catenin mutations. Hepatology 2003, 37:528-533

15. Ovejero C, Cavard C, Périanin A, Hakvoort T, Vermeulen J, Godard C, Fabre M, Chafey P, Suzuki K, Romagnolo B, Yamagoe S, Perret C: Identification of the leukocyte cell-derived chemotaxin 2 as a direct target gene of beta-catenin in the liver. Hepatology 2004, 40: $167-176$

16. Shtutman M, Zhurinsky J, Simcha I, Albanese C, D'Amico M, Pestell R, Ben-Ze'ev A: The cyclin D1 gene is a target of the betacatenin/LEF-1 pathway. Proc Natl Acad Sci U S A 1999, 96: $5522-5527$

17. Nejak-Bowen KN, Zeng G, Tan X, Cieply B, Monga SP: beta-Catenin regulates vitamin $\mathrm{C}$ biosynthesis and cell survival in murine liver. J Biol Chem 2009, 284:28115-28127

18. Lu J, Li XP, Dong Q, Kung HF, He ML: TBX2 and TBX3: the special value for anticancer drug targets. Biochim Biophys Acta 2010, 1806:268-274

19. Monga SP: beta-Catenin signaling and roles in liver homeostasis, injury, and tumorigenesis. Gastroenterology 2015, 148:1294-1310

20. de La Coste A, Romagnolo B, Billuart P, Renard CA, Buendia MA, Soubrane O, Fabre M, Chelly J, Beldjord C, Kahn A, Perret C: Somatic mutations of the beta-catenin gene are frequent in mouse and human hepatocellular carcinomas. Proc Natl Acad Sci U S A 1998, 95:8847-8851

21. Huang H, Fujii H, Sankila A, Mahler-Araujo BM, Matsuda M, Cathomas G, Ohgaki H: beta-Catenin mutations are frequent in human hepatocellular carcinomas associated with hepatitis $\mathrm{C}$ virus infection. Am J Pathol 1999, 155:1795-1801

22. Kondo Y, Kanai Y, Sakamoto M, Genda T, Mizokami M, Ueda R, Hirohashi S: beta-Catenin accumulation and mutation of exon 3 of the beta-catenin gene in hepatocellular carcinoma. Jpn J Cancer Res 1999, 90:1301-1309

23. Legoix P, Bluteau O, Bayer J, Perret C, Balabaud C, Belghiti J, Franco D, Thomas G, Laurent-Puig P, Zucman-Rossi J: beta-Catenin mutations in hepatocellular carcinoma correlate with a low rate of loss of heterozygosity. Oncogene 1999, 18:4044-4046

24. Nhieu JT, Renard CA, Wei Y, Cherqui D, Zafrani ES, Buendia MA: Nuclear accumulation of mutated beta-catenin in hepatocellular carcinoma is associated with increased cell proliferation. Am J Pathol 1999, 155:703-710

25. Hsu HC, Jeng YM, Mao TL, Chu JS, Lai PL, Peng SY: beta-Catenin mutations are associated with a subset of low-stage hepatocellular carcinoma negative for hepatitis B virus and with favorable prognosis. Am J Pathol 2000, 157:763-770 
26. Devereux TR, Stern MC, Flake GP, Yu MC, Zhang ZQ, London SJ, Taylor JA: CTNNB1 mutations and beta-catenin protein accumulation in human hepatocellular carcinomas associated with high exposure to aflatoxin B1. Mol Carcinog 2001, 31:68-73

27. Wong CM, Fan ST, Ng IO: beta-Catenin mutation and overexpression in hepatocellular carcinoma: clinicopathologic and prognostic significance. Cancer 2001, 92:136-145

28. Taniguchi K, Roberts LR, Aderca IN, Dong X, Qian C, Murphy LM, Nagorney DM, Burgart LJ, Roche PC, Smith DI, Ross JA, Liu W: Mutational spectrum of beta-catenin, AXIN1, and AXIN2 in hepatocellular carcinomas and hepatoblastomas. Oncogene 2002, 21: 4863-4871

29. Zucman-Rossi J, Benhamouche S, Godard C, Boyault S, Grimber G, Balabaud C, Cunha AS, Bioulac-Sage P, Perret C: Differential effects of inactivated Axin1 and activated beta-catenin mutations in human hepatocellular carcinomas. Oncogene 2007, 26:774-780

30. Tzameli I, Pissios P, Schuetz EG, Moore DD: The xenobiotic compound 1,4-bis[2-(3,5-dichloropyridiloxy)]benzene is an agonist ligand for the nuclear receptor CAR. Mol Cell Biol 2000, 20:2951-2958

31. Dragani TA, Manenti G, Galliani G, Della Porta G: Promoting effects of 1,4-bis[2-(3,5-dichloropyridyloxy)]benzene in mouse hepatocarcinogenesis. Carcinogenesis 1985, 6:225-228

32. Columbano A, Ledda-Columbano GM, Pibiri M, Piga R, Shinozuka H, De Luca V, Cerignoli F, Tripodi M: Increased expression of c-fos, c-jun and LRF-1 is not required for in vivo priming of hepatocytes by the mitogen TCPOBOP. Oncogene 1997, $14: 857-863$

33. Diwan BA, Lubet RA, Ward JM, Hrabie JA, Rice JM: Tumor-promoting and hepatocarcinogenic effects of 1,4-bis[2-(3,5-dichloropyridyloxy)]benzene (TCPOBOP) in DBA/2NCr and C57BL/6 $\mathrm{NCr}$ mice and an apparent promoting effect on nasal cavity tumors but not on hepatocellular tumors in $\mathrm{F} 344 / \mathrm{NCr}$ rats initiated with N-nitrosodiethylamine. Carcinogenesis 1992, 13:1893-1901

34. Huang W, Zhang J, Washington M, Liu J, Parant JM, Lozano G, Moore DD: Xenobiotic stress induces hepatomegaly and liver tumors via the nuclear receptor constitutive androstane receptor. Mol Endocrinol 2005, 19:1646-1653

35. Mattu S, Fornari F, Quagliata L, Perra A, Angioni MM, Petrelli A, Menegon S, Morandi A, Chiarugi P, Ledda-Columbano GM, Gramantieri L, Terracciano L, Giordano S, Columbano A: The metabolic gene $\mathrm{HAO} 2$ is downregulated in hepatocellular carcinoma and predicts metastasis and poor survival. J Hepatol 2016, 64: 891-898

36. Dragani TA, Manenti G, Della Porta G: Genetic susceptibility to murine hepatocarcinogenesis is associated with high growth rate of NDEA-initiated hepatocytes. J Cancer Res Clin Oncol 1987, 113: 223-229

37. Kowalik MA, Saliba C, Pibiri M, Perra A, Ledda-Columbano GM, Sarotto I, Ghiso E, Giordano S, Columbano A: Yes-associated protein regulation of adaptive liver enlargement and hepatocellular carcinoma development in mice. Hepatology 2011, 53:2086-2096

38. Aydinlik H, Nguyen TD, Moennikes O, Buchmann A, Schwarz M: Selective pressure during tumor promotion by phenobarbital leads to clonal outgrowth of beta-catenin-mutated mouse liver tumors. Oncogene 2001, 20:7812-7816

39. Committee for the Update of the Guide for the Care and Use of Laboratory Animals; National Research Council: Guide for the Care and Use of Laboratory Animals: Eighth Edition. Washington, DC, National Academies Press, 2011

40. Petrelli A, Perra A, Cora D, Sulas P, Menegon S, Manca C, Migliore C, Kowalik MA, Ledda-Columbano GM, Giordano S, Columbano A: MicroRNA/gene profiling unveils early molecular changes and nuclear factor erythroid related factor 2 (NRF2) activation in a rat model recapitulating human hepatocellular carcinoma (HCC). Hepatology 2014, 59:228-241

41. Lee JS, Thorgeirsson SS: Genome-scale profiling of gene expression in hepatocellular carcinoma: classification, survival prediction, and identification of therapeutic targets. Gastroenterology 2004, 127(5 suppl 1):S51-S55

42. Kawamoto T, Sueyoshi T, Zelko I, Moore R, Washburn K, Negishi M: Phenobarbital-responsive nuclear translocation of the receptor CAR in induction of the CYP2B gene. Mol Cell Biol 1999, 19:6318-6322

43. Columbano A, Ledda-Columbano GM, Pibiri M, Cossu C, Menegazzi M, Moore DD, Huang W, Tian J, Locker J: Gadd45beta is induced through a CAR-dependent, TNF-independent pathway in murine liver hyperplasia. Hepatology 2005, 42:1118-1126

44. Rebouissou S, Franconi A, Calderaro J, Letouzé E, Imbeaud S, Pilati C, Nault JC, Couchy G, Laurent A, Balabaud C, BioulacSage P, Zucman-Rossi J: Genotype-phenotype correlation of CTNNB1 mutations reveals different beta-catenin activity associated with liver tumor progression. Hepatology 2016, 64:2047-2061

45. Lee JS, Chu IS, Mikaelyan A, Calvisi DF, Heo J, Reddy JK, Thorgeirsson SS: Application of comparative functional genomics to identify best-fit mouse models to study human cancer. Nat Genet 2004, 36:1306-1311

46. Rao MS, Kokkinakis DM, Subbarao V, Reddy JK: Peroxisome proliferator-induced hepatocarcinogenesis: levels of activating and detoxifying enzymes in hepatocellular carcinomas induced by ciprofibrate. Carcinogenesis 1987, 8:19-23

47. Dong B, Lee JS, Park YY, Yang F, Xu G, Huang W, Finegold MJ, Moore DD: Activating CAR and beta-catenin induces uncontrolled liver growth and tumorigenesis. Nat Commun 2015, 6:5944

48. Laskin DL, Robertson FM, Pilaro AM, Laskin JD: Activation of liver macrophages following phenobarbital treatment of rats. Hepatology 1988, 8:1051-1055

49. Okabe H, Kinoshita H, Imai K, Nakagawa S, Higashi T, Arima K, Uchiyama H, Ikegami T, Harimoto N, Itoh S, Ishiko T, Yoshizumi T, Beppu T, Monga SP, Baba H, Maehara Y: Diverse basis of betacatenin activation in human hepatocellular carcinoma: implications in biology and prognosis. PLoS One 2016, 11:e0152695

50. Loeppen S, Schneider D, Gaunitz F, Gebhardt R, Kurek R, Buchmann A, Schwarz M: Overexpression of glutamine synthetase is associated with beta-catenin-mutations in mouse liver tumors during promotion of hepatocarcinogenesis by phenobarbital. Cancer Res 2002, 62:5685-5688

51. Calvisi DF, Factor VM, Ladu S, Conner EA, Thorgeirsson SS: Disruption of beta-catenin pathway or genomic instability define two distinct categories of liver cancer in transgenic mice. Gastroenterology 2004, 126:1374-1386

52. Rignall B, Braeuning A, Buchmann A, Schwarz M: Tumor formation in liver of conditional beta-catenin-deficient mice exposed to a diethylnitrosamine/phenobarbital tumor promotion regimen. Carcinogenesis $2011,32: 52-57$ 